\title{
Electrostatic Microshutter Arrays
}

\author{
M. J. Li ${ }^{\text {a }}$, A.D. Brown ${ }^{a}$, D. E. Burns ${ }^{\text {b }}$, D. P. Kelly ${ }^{\text {a }}$, K. Kim ${ }^{\text {c }}$ A. S. Kutyrev ${ }^{\text {, }}$ \\ S. R. McCandliss ${ }^{d}$, S. H. Moseley ${ }^{a}$, V. Mikula ${ }^{\mathrm{e}}$, L. H. Oh ${ }^{\mathrm{f}}$ \\ ${ }^{a}$ Goddard Space Flight Center, Greenbelt, MD 20771, USA \\ b NASA Langley Research Center, Hampton, VA 23681, USA \\ ${ }^{\mathrm{c}}$ University of Maryland, College Park, MD 20742 USA \\ d Johns Hopkins University, Baltimore, MD, 20770, USA \\ ${ }^{e}$ The Catholic University of America, Washington D.C. 20064, USA \\ ${ }^{\mathrm{f}}$ SGT Inc., Greenbelt, MD 20770, USA
}

\begin{abstract}
Based on the Microshutter Array (MSA) subsystems developed at NASA Goddard Space Flight Center (GSFC) for the James Webb Space Telescope (JWST), Next Generation Microshutter Array (NGMSA) has been developed to be used as multi-object selectors for future telescopes in space applications. Microshutter arrays function as transmission devices. Selected shutters fully open at 90 degrees permitting income light going through, while the rest of shutters keep closed. The programmable microshutter open and close make the device perform as a multi object selector that can be used on space telescopes. Utilizing a multi object selector, the telescope efficiency can be increased to 100 times or more. Like JWST MSAs, NGMSA features torsion hinges, light shields, front and back electrodes for shutter actuation, latch, and closing. The difference is that JWST MSA utilized the magnetic actuation while NGMSA the electrostatic actuation.
\end{abstract}

\section{KEYWORDS}

micro-optics, near infrared, space telescope, packaging, microshutter, MEMS, multi-object spectroscopy.

\section{INTRODUCTION}

A multi object selector (MOS) is an array of elements that can direct light. A MOS system will enable telescopes to simultaneously obtain spectra from $>100$ targets, which, in turn, increases instrument efficiency one-hundred fold or more. The candidates of MOS in recent years are often MEMS micromirror arrays and microshutter arrays. Micromirrors like in the Digital Micromirror Devices (DMD) can be tilted in a range of angles which redirect the incoming light according to the tilting angle $^{1}$, while microshtters are shutters that can open 90 degrees allowing light transmitting through. The former has the advantage of high fill factor because all driving components can be made hidden underneath the mirrors, while the latter achieves high contrast due to the nature of transmission light. Developments have been made to modify micromirror arrays that can function in cryogenic temperatures, suitable for space applications ${ }^{2}$.

Because of the high contrast, microshutter arrays was selected as the MOS for the Near-IR Spectrometer (NIRSpec) on JWST, which represents one of the three major innovations on the JWST that is scheduled to be launched in 2018 as the successor to the Hubble Space Telescope. The Near-IR Spectrometor (NIRSpec) is one of the four major instruments carried by $\mathrm{JWST}^{3}$, having the microshutter subsystem on the focal plane to select desired galaxies and stars. The NIRSpec instrument was built-up by a collaboration which include NASA, the European Space Agency (ESA), and the Canadian Space Agency (CSA). The JWST launch is built for five years and targeting ten years of service in space. It is demonstrated in Fig. 1 how a MSA is used as a multiple object selector in deep space. The MSAs empower the

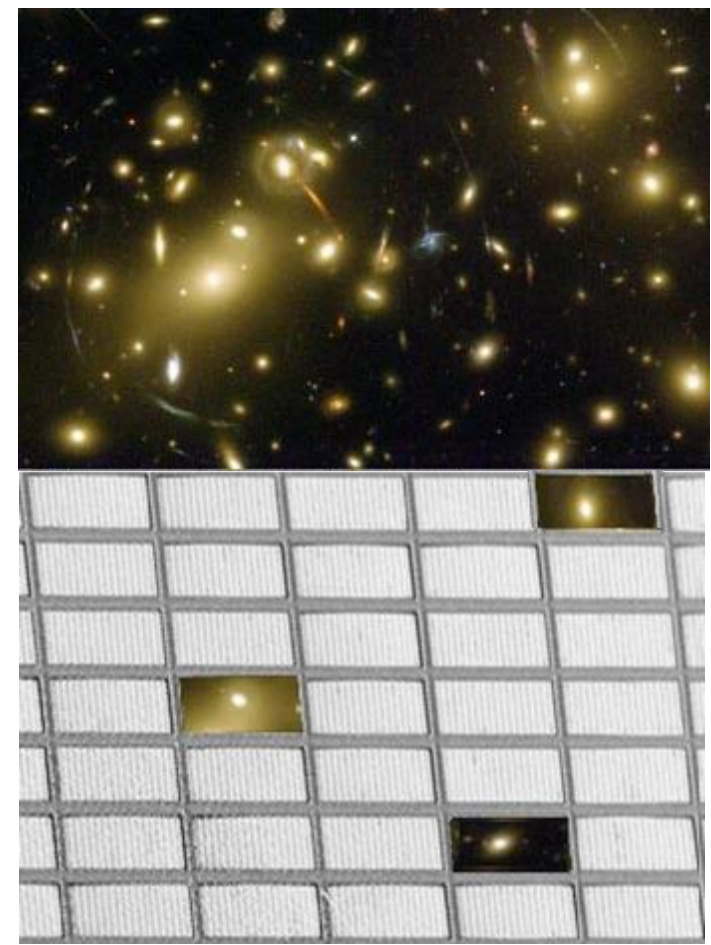

Figure 1 Demonstration of using a microshutter array as an aperture (bottom) to select multiple objects from the sky (top).

NIRSpec instrument to simultaneously collect spectra from more than 100 targets, and therefore increases the instrument efficiency by 100 times or more. The MSA technology is the first major MEMS devices serving observation missions in space.

A wide range of future flight missions will greatly benefit from a multi-object field selector. Scientists are keen to employ multi-object spectroscopy for large-format spectroscopy beyond JWST. The field selectors required for these missions will be much larger than that on JWST. We began to design and develop a scalable field selector, NGMSA, that can be integrated into varieties of large 
format space telescopes, like the Large UV/Optical/Infrared Surveyor (LUVOIR) and the Habitable Exoplanet Imaging Mission (HabEx) .

\section{NGMSA DESIGN}

NGMSA design inherits JWST MSA design in various aspects. JWST MSA was designed to meet the scientific requirement for the JWST mission. The major scientific requirements for the MSA subsystem include ${ }^{4}$ : Random access addressing, -- it is required that the shutters can be opened regardless of their location within the array and that at least 100 objects can be simultaneously selected; Field of View (3.4' x 3.4'); resolution (0.06" x 0.06 "); Contrast >10,000; Life Time 9.4 x $10^{4}$ cycles with minimal failures; and Operating Environment, operate in the JWST test and operation environments, including the entire $300 \mathrm{~K}$ to $35 \mathrm{~K}$ temperature range, the power dissipation of $40 \mathrm{~mW}$ average (at $35 \mathrm{~K}$ ), a volume fitting the envelope in NIRSpec instrument, and a mass of $10 \mathrm{~kg}$, a lifetime radiation dose of $48 \mathrm{kRad}$, To meet the requirements, the MSA flight concept consists of a MEMS mosaic of $2 \times 2$ format of four fully addressable $365 \times 171$ arrays fitting in the JWST optical path at the focal plane. The pixel size is $\sim 100 \times 200 \mu \mathrm{m}$. The MSA system has a mechanism of magnetic actuation and electrostatic latching and addressing 5 . A linear permanent magnet aligned with the shutter rows is positioned on the top of a flipped up-side-down array, as demonstrated in Fig. 2, and

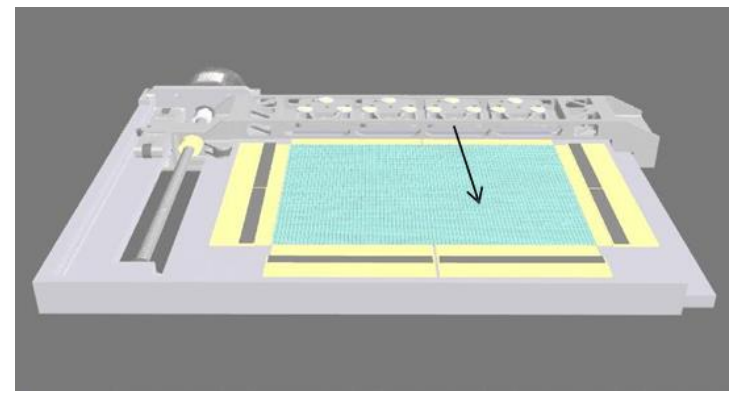

Figure 2 Schematic of a model for the JWST MSA actuation mechanism. A linear magnet sweeps in $Y$ direction across the array.

sweeps across the array in a direction parallel to shutter rows (moving out of the page). As the magnet sweeps across the array, sequential rows of shutters are rotated from their natural horizontal orientation to a vertical open position, where they can be latched to the row electrodes on the sidewalls by a DC bias, typically $35 \mathrm{~V}$. As the bias is removed or insufficient, shutters return to their horizontal closed position upon an elastic restoring force from a torsion flexure on the shutters. The unit cell of NGMSA looks similar to JWST MSA that will be discussed in next section.

The design of NGMSA is based on JWST MSA with a major modification, the actuation mechanism. In order to cover large field of view for future telescopes that may be 50 times or more than JWST's field of view, microshutter arrays have to be made much larger and have to be stitched to form a mosaic. A permanent magnet will not be practical anymore. To remove the magnet and its assecerries like the magnet arm and the step motors, electrostatic actuation is

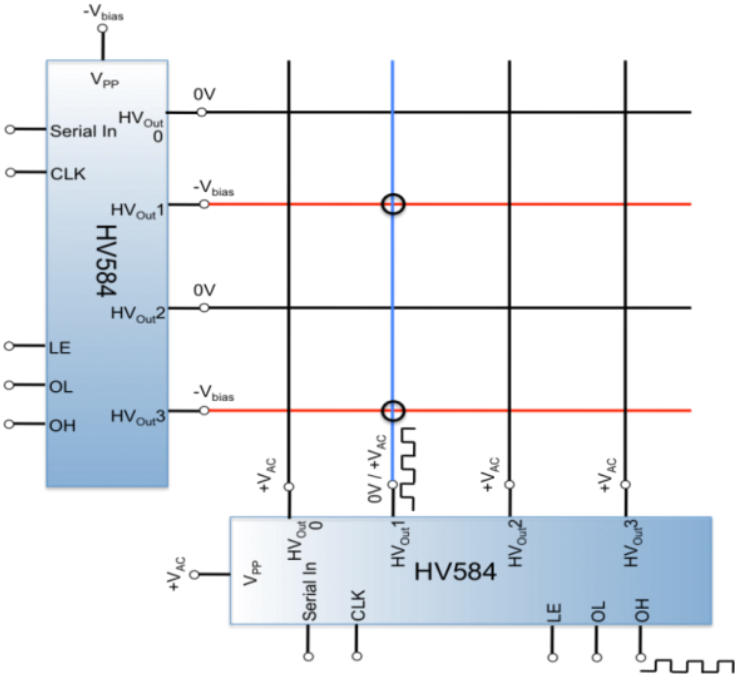

Figure 3 Electrostatic actuation and cross-point 2-D addressing mechanism.

adopted for NGMSA. As illustrated in Figure 3, electrical pulses are applied on the selected shutter column that open the shutter $~ 90$ degrees, and a DC bias applied on the back walls of a selected row catches the shutter and latch it on the back wall. Conductive thin films are tailored on the top of shutters as column electrodes and on the sidewalls in shutter frame windows as row electrodes, respectively. The 2-D addressing method in JWST MSA is adopted for use in NGMSA. IC drivers select the desired columns and rows of shutters to be biased. The shutters at the cross points are kept open and the rest of the shutters kept closed. The difference is shutters are no longer opened by a magnetic force but an electrical pulse.

\section{NGMSA FABRICATION}

NGMSA arrays are fabricated from 4" Silicon-Oxide-Insulation (SOI) wafers. Individual shutters are close-packed silicon nitride membranes with a pixel size approximately equal to $100 \mu \mathrm{m} \mathrm{x}$

\section{INHWHAHANII}

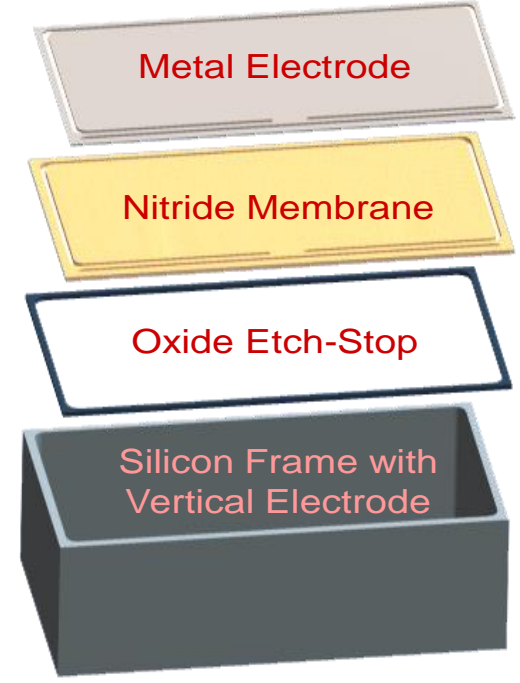

Figure 4 Schematic of unit cell of NGMSA shutter array. 
$200 \mu \mathrm{m}$. The unit cell of microshutters is structured as shown in Figure 4. Shutters are patterned with a fine torsion flexure that connects to the frame permitting shutters to open a full 90 degrees with a minimal amount of mechanical stress. silicon nitride is utilized as the shutter blade materials because of its excellent mechanical and thermal mechanical performances. Aluminum thin film is deposited on the top of shutter blade for two purposes: 1) used as the column metal electrode for electrical biases; and 2) used as a light blocker. A metal nitride thin film is also deposited on the shutter blades and patterned into stripes to reinforce the shutter blades in reducing shutter blade bowing. Aluminum thin film is also deposited on the back wall of the shutter windows as the row metal electrodes for electrical biases. A close-look of fabricated microshutter array is shown in Figure 5 with the view of both front side and back side.

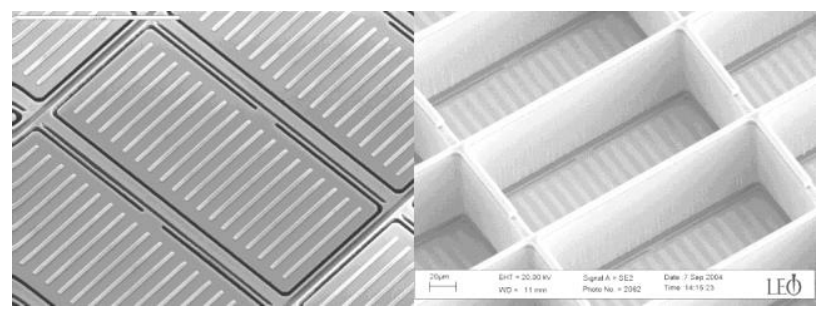

Figure 5 SEM images of the front side (left) and backside (right) of a $64 \times 128$ microshutter array with zoom-in images of shutter cells.

In order to prevent light from leaking around the perimeter of the shutters, light shields are fabricated on to the surrounding frame of each shutter to cover the gaps between the shutters and the frame, as shown in Fig. 6 . Light shields are extend around the perimeter of the microshutters with a $20 \mu \mathrm{m}$ width in the long dimensions of the shutters and a $5 \mu \mathrm{m}$ width in the short dimension. The design was requested by a sounding rocket mission: the Far-UV Off-circle Roland Telescope for imaging and spectroscope (FORTIS) ${ }^{6}$. Sub- micron bumps and micro-ribs are tailored on light shields and back walls, respectively, to prevent stiction. The light shields on the long dimensions of NGMSA shutters are required wider than the JWST MSA, because the former is for UV applications and the latter for IR applications.

The torsion bar thickness was reduced to lessen the torsion bar stiffness and therefore to reduce the voltages for electrostatic actuation. Our COMSOL simulation suggested a reduction in the torsion bar thickness by $50 \%$ in order to reduce the voltages to $\sim 100 \mathrm{~V}$ for the DC pulsed actuation. NGMSA arrays are fabricated with a silicon nitride thickness of $250 \mathrm{~nm}$, the half of the thickness of previous shutters made for JWST. Using a micro-mechanical testing system with a 0.1 micro-Newton accuracy, we measured force vs. deflection data from the NGMSA shutters and previous fabricated shutters as comparison $^{7}$. The testing results indicated that the NGMSA shutter torsion bars stiffness was reduced to one fourth of the previous shutters. The torsion bar thickness

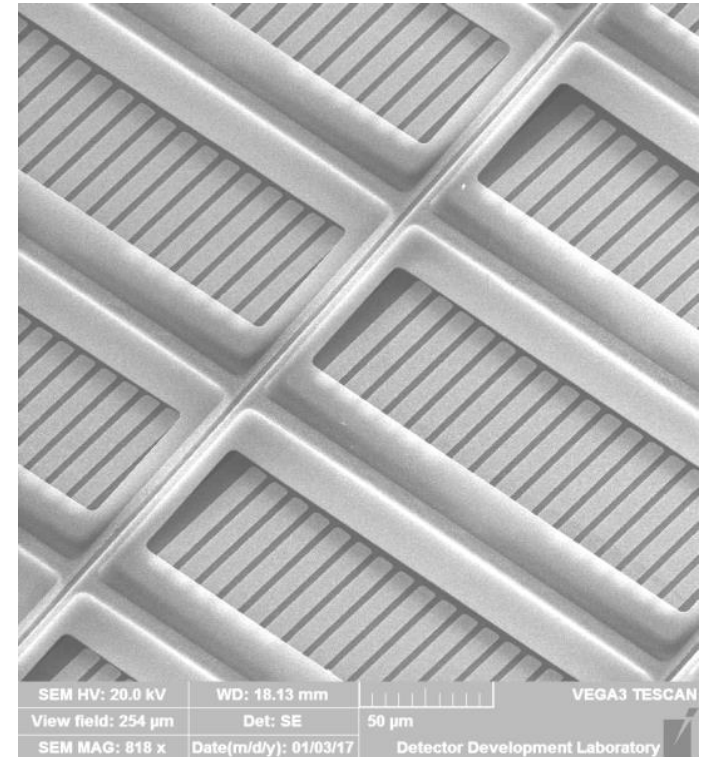

Figure 6 SEM images of microshutters with light shields that prevent light going through the gaps between shutter blade and the frame surrounding the shutters.

reduction is the key to achieve low-voltage actuation of microshutters.

The Atomic-Layer-Deposition (ALD) $\mathrm{Al}_{2} \mathrm{O}_{3}$ is now deposited in a plasma environment that replaced the E-beam deposition ${ }^{8}$. The deposition at the room temperature prevents over-heating of wax that is used to bond the shutter wafer to a handle wafer. The $\mathrm{ALD} \mathrm{Al} \mathrm{O}_{2} \mathrm{O}_{3}$ is a conformal coating that covers everywhere on the back of the microshutter array, not only on the back walls where it supposed to be but also on the backside of shutters that prevent shutters from open. After aluminum deposition on the back walls on the top of the $\mathrm{ALD} \mathrm{Al}_{2} \mathrm{O}_{3}$, a sacrificial silicon oxide is coated to cover the metal, and then the ALD $\mathrm{Al}_{2} \mathrm{O}_{3}$ that covered the back side of shutters can be removed from a XeF2 etch. This modification makes NGMSA with electrical insulation of Gohms between the vertical electrodes and the silicon substrate, a critical breakthrough in microshutter fabrication history.

An antistiction coating was applied on NGMSA as a conformal coating. Stiction is one of the main concerns for the operation of any MEMS devices. When microstructures come into contact with the walls or other

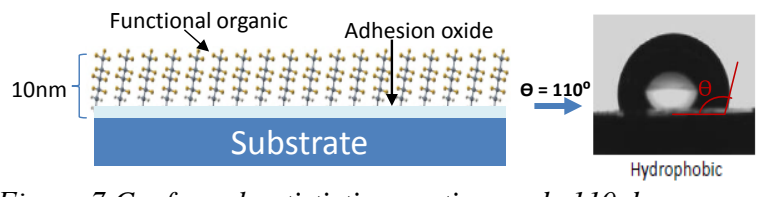

Figure 7 Conformal antistiction coating made 110 degree contact angle on NGMSA surface.

neighboring structures (e.g. light shields), an adhesion caused by capillary, electrostatic discharge, or Van der Waals forces causes stiction and failure to operate (either to the shutter open or to the shutter closed position). The problem wasn't fully resolved in the previous microshutter array development due to the schedule constraints. A coating developed by the Integrated Surface Technologies (IST) Inc, CA, forms a 
10 nanometer-thick composite layer with the oxide side attached to the surface to be coated and the organic side standing out as shown in Figure 7. The organic monolayer makes the array surfaces hydrophobic and can prevent microshutters from sticking to either their light shields or back walls. We applied and tested the antistiction conformal coating on NGMSA arrays and confirmed that the coating prevents shutter stiction.

\section{NGMSA ACTUATION AND 2-D ADDRESSING}

Lab facilities has been established to conduct NGMSA functional testing. Figure 8 shows an 128x64 NGMSA array in a vacuum chamber for actuation tests. The DC pulsed actuation and 2-D addressing sequence is shown in

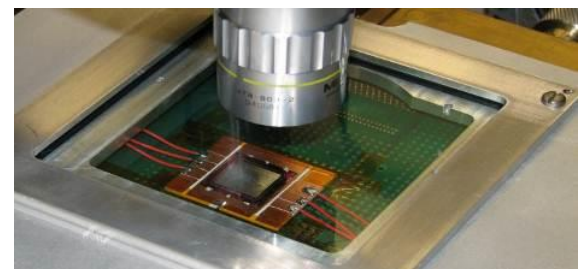

Figure 8 A NGMSA array in a high vacuum chamber for functional tests.

Figure 9. Again, only selected shutters were latched by applying $-25 \mathrm{~V}$ DC to the back electrodes of shutters in two selected columns and 100V DC, $200 \mu \mathrm{sec}$ pulses to the front electrodes of shutters in two selected rows ${ }^{7}$. The $100 \mathrm{~V}$ pulse imparts enough energy to swing the shutters in the rows partly open. The shutters were swung sufficiently open that shutters with DC potentials applied to their back electrodes were able to latch. Continuing to apply the DC potential to the back electrodes held the shutters open. Then the DC potential was selectively removed from the

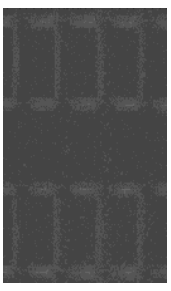

$-25 \mathrm{~V}$ DC on columns one and three

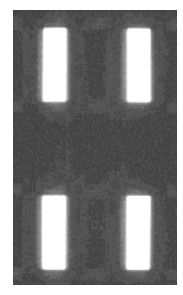

$100 \mathrm{~V}$ pulse to rows one and three

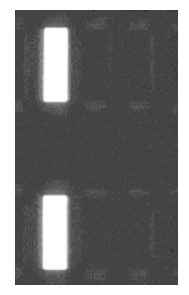

DC potential removed from column one
DC potential removed from column three

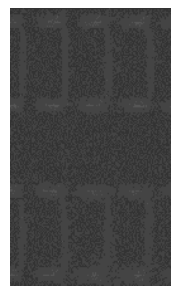

Figure 9The sequence of images demonstrated the electrostatic actuation and 2-D addressings.

back electrodes (columns), causing the shutters to close. Using newly identified IC drivers and modified LabView programs, we are now able to make 2-D addressing images in a large area of a NGMSA array.

\section{SUMMARY}

From JWST MSA, we put efforts in developing NGMSA and introduced new elements in the microshutter array, including electrostatic actuation with DC pulse; thinner torsion bar for reducing torsional stiffness and therefore actuation voltage; ALD aluminum oxide the improve shutter electrode insulation three orders; and antistiction coating. Electrostatic NGMSA provides fast actuation and makes shutter arrays scalable. These elements are critical in enabling microshutter arrays covering large field of view for future large-format telescopes.

The team is working with potential end-users of NGMSA to make NGMSA technically ready for telescopes in future flight missions.

\section{ACKNOWLEDGEMENTS}

The NGMSA team likes to express great appreciation to the packaging team at the Code 553 in Goddard Space Flight Center for their long time packaging support. The NGMSA fabrication was conducted in the Detector Development Lab in Code 553. NGMSA releasing is conducted in the Nano Fabrication Facility in the National Institute of Standards and Technologies. The funding support is from NASA Headquarter.

\section{REFERENCES}

[1] L. Hombeck, "Digital Light Processing: A New MEMS-Based Display Technology" , https://www.rose-hulman.edu/ ahmed/DLP\&DMD.p df

[2] S. Waldis, P. Ayyalasomayajula, W. Noell, N.F. de Rooij, and F. Zamkotsian, "Micromirrors for Multiobject Spectroscopy: Large Array Actuation and Cryogenic Compatibility", https://doi.org/10.1109/OMEMS.2007.4373853

[3] James Webb Space Telescope (JWST) NASA, www.jwst.nasa.gov/

[4] S. H. Moseley et. al., "Microshutter Arrays for theJWSTNear-infraredSpectrometer", Proc. SPIEVol.5487,pp. 645-652, 2004.

[5]M.Li,etinvitedpaper"ComplexMEMSDevice:Microshut terArraySystemforSpaceApplications”,Micro(MEMS )and Nanotechnologiesfor Defense andSecurity,Proc. SPIE, 6556,2007.

[6] FORTIS Takes Flight on Mission to Analyze Comet ISON, http://physics-astronomy.jhu.edu/2013/11/27/fortis-ta kes-flight-on-mission-to-analyze-comet-ison/

[7] D. E. Burns, L. H. Oh, M. J. Li, D. P. Kelly, A. S. Kutyrev, and S. H. Moseley, 2-D Electrostatic Actuation of Microshutter Arrays, JOURNAL OF MICROELECTROMECHANICAL SYSTEMS, VOL. 25, NO. 1, FEBRUARY 2016101.

[8] L. Oh, M. Li, D. Kelley, A. Kutyrev, H. Moseley, "Fabrication of electrostatically Actuated Microshutter Arrays", presented at the $15^{\text {th }}$ International Conference on New Actuators, June 13-15, 2016, Bremen, Germany, ACTUATOR2016.

\section{CONTACT}

*M. J. Li, tel: +1-301-2869921; mary.j.li@ nasa.gov 